\title{
The Experience Structure of Organizational Climate in Universities and the Development of Its Measurement: Based on Chinese Context
}

\author{
Xiaofu Pan, Yuanqi Song \\ School of Cultural \& Social Development Studies, Southwest University, Chongqing, China \\ Email:smilepxf@126.com
}

Received October $6^{\text {th }}$, 2013; revised November $9^{\text {th }}, 2013$; accepted December $5^{\text {th }}, 2013$

\begin{abstract}
Copyright @ 2014 Xiaofu Pan, Yuanqi Song. This is an open access article distributed under the Creative Commons Attribution License, which permits unrestricted use, distribution, and reproduction in any medium, provided the original work is properly cited. In accordance of the Creative Commons Attribution License all Copyrights (C) 2014 are reserved for SCIRP and the owner of the intellectual property Xiaofu Pan, Yuanqi Song. All Copyright (c) 2014 are guarded by law and by SCIRP as a guardian.
\end{abstract}

Based upon the theoretical research and educational practice on school organizational climate at home and abroad, the structural factors of organizational climate in universities were theoretically conceived. The deep interview and self-report questionnaires were adopted, and by using the analyses of exploring factors and of verifying factors, the results show that organizational climate in universities had four dimensions, i.e., administrative climate, teaching climate, learning climate and interpersonal climate, with a total of 16 sub-factors. Results show that the scale of organizational climate in university has good reliability and construct validity. It can be used as assessment tools of organization climate in the university.

Keywords: School Organizational Climate; Factor Analysis; Construct Validity; Reliability

\section{Research Background}

Every university has its unique culture. "In some schools between teachers and principals, they get along well and freely with each other, the teachers seem to be smart, and their mind is full of trust and confidence; However, in another school, it's be poles apart; you can find a kind of tense climate from teachers' facial expression, educating students and their behaviors" (Robert, 1987). In some universities, there is a positive climate. To be united, strict, ordered, and efficient with a set of good rules are their distinguishing characteristics. While in some other universities, there is a negative climate. To be disunited, undisciplined, to deal with things casually, and with a low efficiency are always their features. Take the three famous universities in China: the Peking University, the Tsinghua University and the Beijing Normal University for example. The three universities are all located in Beijing, near each other, and they all are the leading universities of the state. Theoretically, there should be little difference among them. However, one will feel quite different from Tsinghua University to Peking University, and still one will have another kind of feeling from Peking University to Beijing Normal University. It's quite easy for a person to find out the varieties of the taste of the campus, the arrangement of the classrooms, the conditions of the libraries and laboratories, the running and walking in the playgrounds, students' clothes, styles of walking, tones of talking, people's attitudes of meeting each other, and even the features of the presidents (Zhu, 1982). In Peking University, they pursuit "patriotism, progress, democracy and science"; the Tsinghua University focuses on "Self-discipline and social commitment"; while the teachers and students in Beijing Normal University follow "To learn to be a master, to conduct as a model”. Of all these, everything is different. These unique characteristics of each university are referred to as the organizational climate of a university. In fact, the climate of organization may be roughly conceived as the "personality" of the organization, that is, climate is to an organization as personality is to an individual (Halpin, 1963). A university's organizational climate is a set of lasting internal psychological features which can distinguish one university from another (Robert, 1975; Hannum \& Tstchannen-Moran, 1988; Pan, 2007).

There are a lot of researches about the organizational climate in schools home and abroad. These researches are mainly focusing on the following three aspects: 1) Some are describing and measuring the degrees of school organizational climate, such as OCDQ $^{1}$ (Halpin, \& Croft, 1963), OCDQRE and OCDQ-RM (Hoy et al.,1991, 1996), OCI (Stern \& Steinhoff, 1963), POS (Likert, 1968), OCSSS (Pan \& Sun, 2002), and so on; 2) Some are studying the relationship between school organizational climate and the organizational effectiveness, such as school effectiveness (Hoy et al., 1990; Gelade, 2003; Van Houtte, 2005; Griffith, 2006), organizational health (Cullen et al.,1999) and student achievement (Hoy \& Hannum, 1997; Dumay, 2009; Yin, 2009), teachers' job satisfaction (Nalcaci, 2012; Pan, 2007), job burnout (Tian \& Li, 2006) and teacher commitment (Riehl \& Sipple, 1996; Zhu \& Chang, 2011); 3) Some others are trying to predict and manipulate school organizational climate, for example, school climate in predicting

${ }^{1}$ OCDQ: Organization Climate Descriptive Questionnaire; OCI: Organization Climate Index; POS: Profile of a School; OCSSS: Organizational Climate for Secondary School Scale. 
school effectiveness (Hoy et al., 1990), school health (Cullen, 1999), school disorder (Gottfredson, 2005), teachers' job satisfaction, mental health (Deng \& Pan, 2006; Pan \& Qin, 2007; Ou \& Pan, 2008) and student achievement (Hoy \& Hannum, 1997; Dumay, 2009; Yin, 2009). Although researches about school organizational climate, especially those of the higher education, just started not long ago, they have received more and more focuses recently as a result of the popularity of organizational psychology and the development of further reform of schools. However, due to the fact that there is a scarcity of materials of theories from abroad and a lack of measuring tools, the scientific and systematic research of school organizational climate is greatly hindered (Shao, 1998). Therefore, this research aims to compile a scale for measuring the organizational climate in higher education in China, in an attempt to provide a relatively scientific, objective and effective scale for the researches concerned.

\section{Initial Constructions and Dimensionality of University Organizational Climate (UOC)}

Methods of interviewing, semantic analysis, category analysis and Delphi Method are applied to construct the categories of university's organizational climate in four steps. First, openended questionnaire and interview were used to quest for the words reflecting university's organizational climate; then, semantic analysis and category analysis were applied to filtrate the words collected so as to find out the key words; thirdly, based on the above theoretical basis (especially, such as OCDQ, OCDQ-RE, OCDQ-RM, OCI, POS and OCSSS ), an initial questionnaire was composed; and finally, a pilot test was carried out, and a formal questionnaire was compiled after factor analysis of the data..

\section{Open-Ended Questionnaire and Interview}

\section{Sampling}

300 persons of teachers, administrators and students in universities in Chongqing in China were chosen by random. And 9 master students majoring in psychology held the interview. Among the 300 questionnaires, there are altogether 268 valid ones.

\section{Interview and Open-Ended Questionnaire}

Through the interview, key words finding, 300 persons of teachers, administrators and students in universities in Chongqing in China were chosen by random. And 9 master students majoring in psychology held the interview, introducing the meanings of Organizational Climate and the categories to measure them, asking them to list some vocabularies to describe the Organizational Climate of a university. The instruction is "Hello! We want to know about the university's organizational climate in this questionnaire. It is known that every university has its unique climate, for example, there is a climate of 'democratic, fair and orderly...' in administration, 'diligent, hard-working...' in learning, 'vivid, active and rigorous...' in teaching and 'nice, helpful and cooperative...' in inter-person relationship" and so on. Please list at least 5 adjectives to describe the organizational climate in your university, and rank them with the most important one at first. Your opinion is very valuable for our research, please do it according to the real situation of your university and your true feelings. Thank you very much for your cooperation!” Then, after the process of abstracting, the high frequencies of words were left and low frequencies and repeated words were left out, and 86 words such as "morale, learning climate, rules" were left. The left 86 words were then categorized with the method of R-type clustering analysis according to their frequencies, and the result showed that they could be categorized into 24 groups in the lower level. The class distance is 0.165 . After that, they were categorized in a higher level, and they could be grouped into 4, and the class distance is 0.068 . The four groups reflect respectively the aspects of administration, teaching, learning and interpersonal relationship. We name them as Administrative Climate, Interpersonal Climate, Teaching Climate and Learning Climate. Thus we got the idea that UOC can be shown in the four aspects of administrating, teaching, learning and interpersonal relationship. Finally, the connotations of each word are defined as shown table in 2, and a theoretical frame work is constructed thereafter.

\section{Factor Abstraction}

Analyze and categorize the information gathered. First, count the times of adjectives chosen by the participants, keep those above 50\%; secondly, 9 master students majoring in psychology analyze and categorize these adjectives, compare their results and keep those adjectives which have been chosen by more than five students; thirdly, generalize the connotations of these chosen adjectives, and finally, Delphi method is used to categorize these terms. The expert panel was composed of 9 master and doctor students majoring in applied psychology. These experts were told to categorize the selected and sorted adjectives further according to their own understanding and list their standards. This process continued until they reached an agreement about the categorization. The researcher gave the questions related with detailed background information and requirements, and then the experts would answer these questions in written form. Put all the answers together into a table, and send it to the experts. There were only the answers, without any other information related to experts. The experts compared the others' judgments and modify their own. After two turns of this process, the experts almost reached an agreement about the standards of categorization, and the result is showed as Table 1.

Thus, the researchers got the categories of the UOC. The researchers defined these standards, and experts put those concepts into related categories. In this way, a detailed structure came into being, as shown in Table 2.

\section{Compiling and Testing of the UOC Scale}

\section{Compiling of the Scale}

Based on the categories in Table 1, for each subcategory, the researchers complied 6 items (statements in this case), which were believed to be the most typical ones to represent the teachers' behavior. There are altogether 96 statements, of which 28 are reverse scoring. There are five choices ranging in degree from "Never, scarcely, sometimes, often and always" following each statement, counted as " $1,2,3,4,5$ " respectively. Take the statement "The departments of the university can do their jobs appropriately and cooperate and help each other" for an example, if you feel that it is often the case, then you choose "often", and this choice is counted 4. By way of sifting, there were 80 
Table 1.

Values of the $3^{\text {rd }}$ delphi method.

\begin{tabular}{|c|c|c|c|c|c|c|c|}
\hline \multirow[t]{3}{*}{ ITEMS EVALUATED } & \multicolumn{5}{|c|}{ VALUE RANGES } & \multirow{3}{*}{$\begin{array}{c}\text { MEANS } \\
\text { (M) }\end{array}$} & \multirow{3}{*}{$\begin{array}{c}\text { FILTER VALUE } \\
\text { (M > 4, } \\
\text { AGREED) }\end{array}$} \\
\hline & Completely agreed & agreed & uncertain & disagreed & Completely disagreed & & \\
\hline & 5 & 4 & 3 & 2 & 1 & & \\
\hline Teaching & 7 & 2 & & & & 4.78 & $\sqrt{ }$ \\
\hline Learning & 7 & 1 & 1 & & & 4.67 & $\sqrt{ }$ \\
\hline Campus culture & 3 & 3 & 1 & 1 & 1 & 3.67 & $\times$ \\
\hline Physical environment & 3 & 1 & 2 & 2 & 1 & 3.33 & $\times$ \\
\hline
\end{tabular}

Table 2.

UOC’s internal structure’s categories and their definitions.

\section{Administrative Climate (AC)}

1) Administrative order (AO): It is a behavioral relationship formed between unit members based on organizational structure’s system and its management functions. Are there rules for people to follow? Do people act as they are supposed? Are those organizational behaviors planned, ordered? 2) Administrative style (AS): This refers to managers' approaches to management. And it mainly displays itself in one point on the continuum of "Democracy-autocracy, seriousness-flexibility and openness-closed".

3) Administrative morality (AM): This means that whether the managers can be fair, just and open in the process of doing their duties.

4) Administrative efficiency (AE): Do managers possess good qualities and skills to manage? What is the efficiency of managing?

\section{Interpersonal Climate (IC)}

1) Interpersonal action (IAc): This refers to the relationship between persons. It shows itself as whether they are united and help each other in their work.

2) Interpersonal harmony (IH): Interpersonal harmony is a feeling of the environment, such as a harmonious and peaceful interpersonal relationship.

3) Interpersonal attitude (IAt): This is a tendency of recognizing and attracting each other, such as friendly, kind and enthusiastic to each other.

4) Interpersonal distance (ID): This is what persons perceive about the remoteness and closeness of the interpersonal relationship.

\section{Teaching Climate (TC)}

1) Teaching attitude (TAt): This refers to what the teachers think of teaching and the actions taken accordingly. It displays in that whether teachers deal with every aspect of the teaching rigorously, seriously and conscientiously.

2) Teaching arts (TAr): Teaching arts means the methods and measures taken by the teachers in the teaching process and the flexibility, craftsmanship and wisdom displayed in teaching.

3) Teaching style (TS): A stable and habitual teaching model formed in the long time of teaching, such as rigidness or flexibility.

4) Teaching result (TR): What are the quality and effectiveness and efficiency of teaching.

\section{Learning Climate (LC)}

1) Learning attitude (LA): Whether people are active, persevere and diligent in learning.

2) Learning stress (LS): A sense of the learning, whether it is stressed, free and active or not.

3) Learning method (LM): Whether a cooperative learning style is applied and whether a creative spirit is shown.

4) Learning result (LR): What are the quality and effectiveness and efficiency of learning.

items in the pilot questionnaire. In order to tell whether the participants are doing their job or not, several items expressing the same meaning by using different structures have been scattered in the questionnaire.

\section{Pilot Test}

\section{Sample and test}

350 stuff members were chosen randomly in 12 universities in Chongqing (the ratio of teachers, assistants and administrators is 4:3:3), and they answered the questionnaire. 306 valid questionnaires were gathered.

\section{Exploratory Factor Analysis}

The methods of the principal component method and Direct Oblimin are used to analyze the factors, and the result is:

1) $\mathrm{KMO}$ and Bartlett's Test of Sphericity, Chi-Square = 3030.60, $p<0.001$; there are 4 marked turning points in Scree plot, and this shows that the data are suitable for factor analysis.

2) Through the method of the principal component method and Direct Oblimin to analyze factors, we got the result of 19 items belonging to one factor, they all reflect the character of managing. Therefore, this factor is named as Administrative Climate (AC). These items are 1, 2, 3, 4, 5, 6, 7, 8, 9, 10, 11, 12, $13,15,16,17,18,19$ and 20 . Of the 19 items, the Eigenvalue is 9.72, factor loading is $0.42-0.83$, the contribution rate is $13.4 \%$. Items of 22, 23, 24, 25, 26, 28, 29, 30, 31, 32, 33, 34, 35, $36,37,38,39$, 40 belong to one factor, and they reflect characters of Interpersonal Climate IC). Of these 18 items, the Eigenvalue is 6.55 , factor loading from 0.41 to 0.86 , the contribution rate is $12.7 \%$ 。 Another 17 items of 41, 42, 43, 44, 45, 46, 47, $48,49,50,51,52,53,55,56,58$ and 60 gathers around one factor, showing characteristics of teaching, and is named as Teaching Climate (TC). Of the 17 items, the Eigenvalue is 5.61, factor loading is $0.44-0.87$, the contribution rate is $9.5 \%$. The last group of 17 items gathers around one factor, showing the 
characters of learning, and it is named as Learning Climate (LC). These items are 61, 62, 63, 64, 65, 66, 67, 68, 69, 70, 71, $72,73,75,77,78$ and 80 . Of the 17 items, the Eigenvalue is 3.65 , factor loading is $0.41-0.87$, the contribution rate is $5.7 \%$.

(3) There are 9 items of 14, 21, 27, 54, 57, 59, 74, 76 and 79 deeted, due to their low and dispersed factor loading. The final questionnaire is composed of 71 items.

\section{Testing of Validity and Reliability of UOC Scale \\ Testing of Validity and Reliability of the Whole Scale \\ Sampling}

16 universities were chosen from Chinese five districts: east, west, south, north and the central ones. They were: Inner Mongolia University of Finance and Economics, Wuhan University of Technology, Hunan Normal University, Changsha University of Science and Technology, Xiangnan University, Guangxi University, Guangxi Normal University, Southwest University, Chongqing Normal University, Chongqing College of Education, Yunnan Dali University, Guizhou Normal University, Suzhou University, Jiangsu University of Technology, Zhejiang Taizhou University. Fifty teachers in each university were chosen by random to answer the questionnaire. Among the delivered 800 questionnaires, there are 532 valid ones. There are $46.99 \%$ of males, $53.01 \%$ of females answered the questionnaire. Among them, $40.0 \%$ is postgraduates, $49.4 \%$ is graduates and $10.6 \%$ don't get a degree of graduate. From the aspect of ranks, $9.7 \%$ of them is professors, $25.8 \%$ is associate professors, $34.3 \%$ is lecturers and $30.2 \%$ is assistant professors. $59.1 \%$ of them has worked for less than 10 years, $18.8 \%$ of them has worked for 10 to 20 years, $14.4 \%$ for 20 to 30 years. $7.7 \%$ of them have worked for more than 30 years.

\section{Validity Test}

1) Exploratory Factor Analysis

The methods of the principal component method and Direct Oblimin are used to analyze the factors, and the result is: a) KMO and Bartlett's Test of Sphericity, Chi-Square $=14530.55$, $p<0.001$; there are 4 marked turning points in Scree plot, and this shows that the data are suitable for factor analysis. b) Through the method of the principal component method and Direct Oblimin to analyze factors, the result is shown in Table 3.

Data for Table 3 show that the model of 4 factors is rather good. The values of each factor loading is high in the four categories from the factor analysis, ranging from 0.436 to 0.721 in AC, from 0.408 to 0.623 in TC, from 0.461 to 0.683 in LC and from 0.417 to 0.683 in IC. At the same time, the accumulation of contributions ratio of each category is 79.661, of which AC accounts for $27.887 \%$ of the total variance, IC accounts for $26.491 \%$, TC accounts for $15.216 \%$, and LC accounts for $10.067 \%$. These support that the theoretical factor structure is suitable.

\section{2) Confirmatory Test}

LISREL 8.0 is applied to the Confirmatory Factor Analysis of the internal structural components. The main purpose is to exam its fitting degree. LISREL provides a systemic approach to analyze and study data. It permits researchers to evaluate the measurement and structural parts (the cause and result relationship) at the same time. Fitting degree refers to the degree of conformity of the structural part and the actual measurements. The fitting degree of this study is shown in Table 4.
It is generally believed that if the value of $\chi^{2} / \mathrm{df}$ is small, the fitting degree of the model is good. The fitting degree in this study is acceptably good. If the value of the fitting degree index of RFI, NFI, NNFI, CFI, IFI is approaching 1, and RASEA is smaller than 0.08 , the theoretic assumption of the study is better. The values of RFI, NFI, NNFI, CFI, IFI are all above 0.90, and RASEA $(=0.057)$ is lower than 0.08 in this study. These demonstrate that the fitting degree of the four factors is good, having a good structural validity.

Judging from the above, we can draw the conclusion that UOC is composed of 71 items from the AC, TC, LC and IC four categories and its 16 sub-factors. By way of filtering degree analysis, the indexes of each fitting are feasible, and this further proves that there is good consistence between the theoretical model and the empirical research, therefore, the scale of UOC has a good construct validity.

\section{Reliability Test}

1) The reliability of internal consistency coefficient and splithalf

The test of reliability of the UOC through the reliability of internal consistency coefficient and split-half shows that the internal consistency coefficient (Alpha) is 0.9406, and the reliability of split-half $(\mathrm{r})$ is $0.8369(p<0.01)$. This proves that this scale has a good reliability.

2) Retest of reliability

Two weeks after the test, another 150 stuff members from Chongqing universities were chosen and did the questionnaire again. There were 139 valid questionnaires this time. Using Pearson's correlation analysis to analyze the two tests, we got the following results of the retest reliability: (r): AC is 0.93 , TC is 0.89 , LC is 0.91 , IC is 0.94 , the whole scale is 0.92 ; its significant is $p<0.01$. These demonstrate that the scale has a good reliability.

\section{Validity Test of the Sub Scales}

Next step is to test the validity of the sub scales of AC, LC, TC and IC through the method of factor analysis.

\section{Test of Validity and Reliability of AC}

1) Factor analysis.

First, The methods of the principal component method and Varimax Rotation are used, Chi-Square $=3222.57, p<0.00$; there are 4 marked turning points in Scree plot, and this shows that the data are suitable for factor analysis. Then, the method of the principal component method and Varimax Rotation is used, and the result is shown in Table 5.

Data from Table 5 show that the values of factor loading in AC support the theoretical construction of the scale. The values of factor loading in AO range from 0.494 to 0.765 , accumulate contributory rate is $23.75 \%$; the values of factor loading in AS range from 0.41 to 0.811 , accumulate contributory rate is $38.13 \%$; the values of factor loading in AM range from 0.445 to 0.670 , accumulate contributory rate is $50.46 \%$; the values of factor loading in $\mathrm{AO}$ range from 0.536 to 0.802 , accumulate contributory rate is $59.74 \%$.

2) Test of Confirmatory Factor Analysis

LISREL 8.0 is applied to the Confirmatory Factor Analysis of this scale. And the values of GFI, AGFI, NFI, NNFI, CFI, IFI of the four sub-factors categories of AO, AS, AM and AE are all above 0.90 , and RASEA is smaller than 0.08. These 
Table 3.

Factor analysis of the internal structural categories of UOC $(\mathrm{N}=532)$.

\begin{tabular}{|c|c|c|c|c|c|c|c|}
\hline Item & Factor 1 & Item & Factor 2 & Item & Factor 3 & Item & Factor 4 \\
\hline 16 & 0.721 & 34 & 0.683 & 60 & 0.623 & 62 & 0.683 \\
\hline 17 & 0.720 & 26 & 0.640 & 53 & 0.565 & 63 & 0.654 \\
\hline 9 & 0.707 & 32 & 0.625 & 44 & 0.561 & 67 & 0.597 \\
\hline 5 & 0.658 & 33 & 0.599 & 46 & 0.538 & 64 & 0.568 \\
\hline 19 & 0.621 & 25 & 0.542 & 58 & 0.537 & 70 & 0.563 \\
\hline 12 & 0.605 & 29 & 0.533 & 47 & 0.521 & 78 & 0.543 \\
\hline 1 & 0.562 & 39 & 0.531 & 42 & 0.509 & 61 & 0.539 \\
\hline 18 & 0.556 & 30 & 0.521 & 43 & 0.508 & 65 & 0.538 \\
\hline 4 & 0.553 & 23 & 0.501 & 51 & 0.488 & 66 & 0.533 \\
\hline 2 & 0.529 & 40 & 0.499 & 49 & 0.487 & 69 & 0.519 \\
\hline 13 & 0.528 & 31 & 0.490 & 50 & 0.475 & 68 & 0.513 \\
\hline 20 & 0.501 & 37 & 0.446 & 45 & 0.468 & 72 & 0.508 \\
\hline 8 & 0.501 & 22 & 0.437 & 48 & 0.458 & 80 & 0.502 \\
\hline 10 & 0.484 & 36 & 0.427 & 41 & 0.454 & 73 & 0.499 \\
\hline 3 & 0.472 & 28 & 0.420 & 56 & 0.416 & 77 & 0.468 \\
\hline 15 & 0.462 & 38 & 0.420 & 55 & 0.408 & 71 & 0.461 \\
\hline 7 & 0.462 & 34 & 0.417 & & & & \\
\hline 11 & 0.436 & & & & & & \\
\hline Factors & OC & & IC & & TC & & LC \\
\hline Eigen Value & 8.710 & & 8.393 & & 7.373 & & 7.254 \\
\hline Contribution ratio (CR) \% & 27.887 & & 26.491 & & 15.216 & & 10.067 \\
\hline Accumulation of CR \% & 27.887 & & 54.378 & & 69.594 & & 79.661 \\
\hline
\end{tabular}

Table 4.

Fitting degree of the confirmatory factor analysis of oc in universities.

\begin{tabular}{ccccccc}
\hline$\chi^{2}$ & RMR & RASEA & NFI & IFI & CFI & $\chi^{2} / \mathrm{df}$ \\
\hline UOC Model & 1124.53 & 0.06 & 0.057 & 0.92 & 0.91 & 0.93 \\
\hline
\end{tabular}

Table 5.

Factor analysis of AC subscale.

\begin{tabular}{|c|c|c|c|c|c|c|c|}
\hline Item & Factor 1 & Item & Factor 2 & Item & Factor 3 & Item & Factor 4 \\
\hline 2 & 0.765 & 7 & 0.811 & 11 & 0.670 & 20 & 0.802 \\
\hline 1 & 0.650 & 9 & 0.662 & 13 & 0.578 & 17 & 0.680 \\
\hline 4 & 0.558 & 8 & 0.552 & 15 & 0.445 & 18 & 0.642 \\
\hline 5 & 0.494 & 10 & 0.491 & & & 19 & 0.536 \\
\hline Eigen Value & 4.03 & & 2.44 & & 2.09 & & 1.53 \\
\hline Contribution ratio (CR) \% & 23.75 & & 14.37 & & 12.33 & & 9.00 \\
\hline Accumulation of CR \% & 23.75 & & 38.13 & & 50.46 & & 59.47 \\
\hline
\end{tabular}

demonstrate that the fitting degree of the four factors is good, having a good construct validity.

3) Reliability test

a) The test of reliability of the AC through the reliability of internal consistency coefficient and the reliability of split-half shows that the internal consistency coefficient (Alpha) is 0.937 $(p \vee 0.001)$, and the reliability of split-half $(r)$ is $0.859(p \vee$ $0.01)$. This proves that this scale has a good reliability. b) Retest of reliability. Two weeks after the test, another 150 stuff members from Chongqing universities were chosen and did the questionnaire again. There were 139 valid questionnaires this time. Using Pearson production-moment correlation analysis to analyze the two tests, we got the following results of the retest reliability (r): AO is 0.87 , AS is $0.84, \mathrm{AM}$ is $0.81, \mathrm{AE}$ is 0.88 , the whole scale is 0.89 ; its significant is $p<0.01$. These demonstrate that the questionnaire has a good reliability. 
Test of Validity and Reliability of IC

1) Factor analysis.

First, The methods of the principal component method and Varimax Rotation are used, Chi-Square $=3035.22, p<0.001$; there are 4 marked turning points in Scree plot, and this shows that the data are suitable for factor analysis. Then, the method of the principal component method and Varimax Rotation are used is applied, and the result is shown in Table 6.

Data from Table 6 show that the values of factor loading in IC support the theoretical construction of the scale. The values of factor loading in IAc range from 0.429 to 0.591 , accumulate contributory rate is $18.4 \%$; the values of factor loading in $\mathrm{IH}$ range from 0.459 to 0.857 , accumulate contributory rate is $36.08 \%$; the values of factor loading in IAt range from 0.466 to 0.755 , accumulate contributory rate is $50.60 \%$; the values of factor loading in ID range from 0.442 to 0.835 , accumulate contributory rate is $62.44 \%$.

2) Confirmatory test

LISREL 8.0 is applied to the Confirmatory Factor Analysis of this scale. And the values of GFI, AGFI, NFI, NNFI, CFI, IFI of the four sub categories of IAc, IH, IAt and ID are all above 0.90 , and RASEA is smaller than 0.08. These demonstrate that the fitting degree of the four factors is good, having a good construct validity.

3) Reliability test

a) The test of reliability of the IC through the reliability of internal consistency coefficient and the reliability of split-half shows that the internal consistency coefficient (Alpha) is 0.941 $(p \vee 0.001)$, and the reliability of split-half ( $\mathrm{r})$ is $0.913(p \vee$ $0.01)$. This proves that this scale has a good reliability.

b) Retest of reliability. Two weeks after the test, another 150 stuff members from Chongqing universities were chosen and did the questionnaire again. There were 139 valid questionnaires this time. Using Pearson production-moment correlation analysis to analyze the two tests, we got the following results of the retest reliability (r): IAc is 0.93 , IH is 0.91 , IAt is 0.89 , ID is 0.88 , the whole scale is 0.90 ; its significant is $p<0.01$. These demonstrate that the questionnaire has a good reliability.

\section{Test of Validity and Reliability of TC}

1) Factor analysis.

First, The methods of the principal component method and Varimax Rotation are used, Chi-Square $=3045.00, p<0.001$; there are 4 marked turning points in Scree plot, and this shows that the data are suitable for factor analysis. Then, the method of the principal component method and Varimax Rotation is applied, and the result is shown in Table 7.

Data from Table 7 show that the values of factor loading in AC support the theoretical construction of the scale. The values of factor loading in TAt range from 0.427 to 0.796 , accumulate contributory rate is $16.12 \%$; the values of factor loading in TAr range from 0.441 to 0.796 , accumulate contributory rate is $30.56 \%$; the values of factor loading in TS range from 0.494 to 0.609 , accumulate contributory rate is $52.39 \%$; the values of factor loading in TR range from 0.494 to 0.609 , accumulate contributory rate is $52.39 \%$.

2) Confirmatory test

LISREL 8.0 is applied to the Confirmatory Factor Analysis of this scale. And the values of GFI, AGFI, NFI, NNFI, CFI, IFI of the four sub categories of TAt, TAc, TS and TRD are all above 0.90 , and RASEA is smaller than 0.08. These demonstrate that the fitting degree of the four factors is good, having a good construct validity.

3) Reliability test

a) The test of reliability of the TC through the reliability of internal consistency coefficient and the reliability of split-half shows that the internal consistency coefficient (Alpha) is 0.909 $(p \vee 0.001)$, and the reliability of split-half ( $\mathrm{r})$ is $0.872(p \vee$ $0.01)$. This proves that this scale has a good reliability.

b) Retest of reliability. Two weeks after the test, another 150 stuff members from Chongqing universities were chosen and did the questionnaire again. There were 139 valid questionnaires this time. Using Pearson production-moment correlation analysis to analyze the two tests, we got the following results of the retest reliability (r): TAt is 0.92 , TAr is 0.87 , TS is 0.94 , TR is 0.86 , the whole scale is 0.91 ; significant is $p<0.01$. These demonstrate that the questionnaire has a good reliability.

\section{Test of Validity and Reliability of LC}

1) Factor analysis.

First, The methods of the principal component method and Varimax Rotation are used, Chi-Square $=3091.58, p<0.001$; there are 4 marked turning points in Scree plot, and this shows that the data are suitable for factor analysis. Then, the method of the principal component method and Varimax Rotation is applied, and the result is shown in Table 8.

Data from Table 8 show that the values of factor loading in LC support the theoretical construction of the scale. The values of factor loading in LA range from 0.414 to 0.749 , accumulate contributory rate is $16.66 \%$; the values of factor loading in LS range from 0.608 to 0.767 , accumulate contributory rate is $31.98 \%$; the values of factor loading in LM range from 0.444 to 0.780 , accumulate contributory rate is $44.63 \%$; the values of factor loading in LR range from 0.661 to 0.754 , accumulate contributory rate is $54.63 \%$.

2) Confirmatory test

LISREL 8.0 is applied to the Confirmatory Factor Analysis of this scale. And the values of GFI, AGFI, NFI, NNFI, CFI, IFI of the four sub categories of LA, LS, LM and LR are all above 0.90 , and RASEA is smaller than 0.08. These demonstrate that the fitting degree of the four factors is good, having a good structural validity.

3) Reliability test

a) The test of reliability of the TC through the reliability of internal consistency coefficient and the reliability of split-half shows that the internal consistency coefficient (Alpha) is 0.915 $(p<0.001)$, and the reliability of split-half $(\mathrm{r})$ is 0.893 ( $p<$ $0.01)$. This proves that this scale has a good reliability.

b) Retest of reliability. Two weeks after the test, another 150 stuff members from Chongqing universities were chosen and did the questionnaire again. There were 139 valid questionnaires this time. Using Pearson production-moment correlation analysis to analyze the two tests, we got the following results of the retest reliability (r): LA is 0.93 , LS is $0.94, \mathrm{LM}$ is 0.89 , LR is 0.87 , the whole scale is 0.92 ; significant is $p<0.01$. These demonstrate that the questionnaire has a good reliability.

\section{Application of UOC}

\section{Counting Method}

UOC reflects the perceptions of the whole stuff members of a university, and therefore, the counting should treat the university as a whole, that is to say, to count the sum of each participant as the original. The way to count is shown in the following 
Table 6.

Factor analysis of IC sub-scale.

\begin{tabular}{|c|c|c|c|c|c|c|c|}
\hline Item & Factor 1 & Item & Factor 2 & Item & Factor 3 & Item & Factor 4 \\
\hline 23 & 0.591 & 30 & 0.857 & 35 & 0.755 & 37 & 0.835 \\
\hline 24 & 0.483 & 29 & 0.678 & 33 & 0.774 & 40 & 0.795 \\
\hline 25 & 0.451 & 28 & 0.580 & 31 & 0.558 & 39 & 0.642 \\
\hline \multirow[t]{2}{*}{22} & 0.429 & 26 & 0.459 & 32 & 0.535 & 38 & 0.592 \\
\hline & & & & 34 & 0.466 & 36 & 0.442 \\
\hline Eigen Value & 2.39 & & 2.29 & & 1.88 & & 1.53 \\
\hline Contribution ratio (CR) \% & 18.40 & & 17.67 & & 14.52 & & 11.83 \\
\hline Accumulation of CR \% & 18.40 & & 36.08 & & 50.60 & & 62.44 \\
\hline
\end{tabular}

Table 7.

Factor analysis of TC sub-scale.

\begin{tabular}{|c|c|c|c|c|c|c|c|}
\hline Item & Factor 1 & Item & Factor 2 & Item & Factor 3 & Item & Factor 4 \\
\hline 41 & 0.767 & 48 & 0.796 & 53 & 0.779 & 56 & 0.609 \\
\hline 45 & 0.676 & 49 & 0.681 & 51 & 0.773 & 58 & 0.577 \\
\hline 44 & 0.445 & 50 & 0.539 & 55 & 0.510 & & \\
\hline 43 & 0.427 & 47 & 0.441 & & & & \\
\hline Eigen Value & 3.38 & & 3.03 & & 2.43 & & 2.14 \\
\hline Contribution ratio (CR) \% & 16.12 & & 14.43 & & 11.60 & & 10.22 \\
\hline Accumulation of CR \% & 16.12 & & 30.56 & & 42.17 & & 52.39 \\
\hline
\end{tabular}

Table 8.

Factor analysis of LC sub-scale.

\begin{tabular}{|c|c|c|c|c|c|c|c|}
\hline Item & Factor 1 & Item & Factor 2 & Item & Factor 3 & Item & Factor 4 \\
\hline 64 & 0.749 & 66 & 0.767 & 75 & 0.780 & 80 & 0.754 \\
\hline 63 & 0.552 & 68 & 0.732 & 71 & 0.724 & 78 & 0.661 \\
\hline 65 & 0.500 & 70 & 0.668 & 72 & 0.585 & 77 & 0.661 \\
\hline 62 & 0.448 & 67 & 0.641 & 73 & 0.444 & & \\
\hline 61 & 0.414 & 69 & 0.608 & & & & \\
\hline Eigen Value & 3.33 & & 3.06 & & 2.53 & & 2.00 \\
\hline Contribution ratio (CR) \% & 16.66 & & 15.32 & & 12.64 & & 10.00 \\
\hline Accumulation of CR \% & 16.66 & & 31.98 & & 44.63 & & 54.63 \\
\hline
\end{tabular}

(the number in the brackets are the item numbers, using the value of the item to substitute):

$$
\begin{gathered}
F_{A C}=f_{A S}+f_{A O}+f_{A M}+f_{A E} \\
{\left[f_{A O}=(1+2+3+4+5) / 5 ; f_{A S}=(6+7+8+9+10) / 5 ;\right.} \\
\left.f_{A M}=(11+12+13+15) / 4 ; f_{A E}=(16+17+18+19+20) / 5\right] \\
F_{I C}=f_{I A C}+f_{I H}+f_{I A t}+f_{I D} ; \\
{\left[f_{I A C}=(22+23+24+25) / 4 ; f_{I H}=(26+28+29+30) / 4 ;\right.} \\
f_{I A t}=(31+32+33+34+35) / 5 ; \\
\left.f_{I D}=(36+37+38+39+40) / 5\right]
\end{gathered}
$$

$$
\begin{gathered}
F_{T C}=f_{T A t}+f_{T A r}+f_{T S}+f_{T E} \\
{\left[f_{T A t}=(41+42+43+44+45) / 5 ;\right.} \\
f_{T A r}=(46+47+48+49+50) / 5 ; \\
\left.f_{T S}=(51+52+53+55) / 4 ; f_{T E}=(56+58+60) / 3\right] \\
F_{L O}=f_{L A}+f_{L S}+f_{L M}+f_{L R} \\
{\left[f_{L A}=(61+62+63+64+65) / 5 ;\right.} \\
f_{L S}=(66+67+68+69+70) / 5 ; \\
f_{L M}=(71+72+73+75) / 4 ; \\
\left.f_{L R}=(77+78+80) / 3\right]
\end{gathered}
$$




\section{Standar Dization}

Applying the standardization method of Hoy (1991), the following counting method can be used in this study: 500 is used as the average score of each category of every school, 100 as the dispersion to standardize the scores, and the result is named as SDS. The counting function is: $\operatorname{SDS}=100 \times(\chi-\mathrm{X}) / \mathrm{SD}$ $+500\{\chi$ : score of each factor; $\mathrm{X}$ : the overall average score; SD: population standard deviation $\}$.

$$
\begin{aligned}
& S D S_{A C}=\left(S D S_{A S}+S D S_{A O}+S D S_{A M}+S D S_{A E}\right) / 4 \\
& S D S_{T C}=\left(S D S_{T A t}+S D S_{T A r}+S D S_{T S}+S D S_{T R}\right) / 4 \\
& S D S_{I C}=\left(S D S_{I A C}+S D S_{I H}+S D S_{I A t}+S D S_{I D}\right) / 4 \\
& S D S_{L C}=\left(S D S_{L A t}+S D S_{L S}+S D S_{L M}+S D S_{L R}\right) / 4
\end{aligned}
$$

When the values of $\mathrm{SDS}_{\mathrm{AC}}, \mathrm{SDS}_{\mathrm{TC}}, \mathrm{SDS}_{\mathrm{IC}}, \mathrm{SDS}_{\mathrm{LC}}$ are above 500 , the bigger the number is, the better the organizational climate is in a school. When the values of $\mathrm{SDS}_{\mathrm{AC}}, \mathrm{SDS}_{\mathrm{TC}}$, $\mathrm{SDS}_{\mathrm{IC}}, \mathrm{SDS}_{\mathrm{LC}}$ are below 500 , the smaller the number is, the poorer the organizational climate is in a school.

\section{Conclusion}

This study applies the methods of interviewing, semantic analysis, category analysis and Delphi method to collect and analyze the data, and further to get the theoretical construction of the questionnaire, namely the four main categories of AC, IC, TC, LC and their 16 sub-factor categories. After the exploratory factor analysis of the pilot questionnaire, it shows that the scale composed of 71 items can objectively reflect the real organizational climate in a university. Confirmatory factor analysis strongly supports the theoretical model construction. It demonstrates the good structural validity of the model. Reliability test, either the reliability of internal consistency coefficient or the reliability of split-half, or the retest of reliability, shows that the self-compiled scale has a very good reliability, and it is a good measuring scale.

Although there are good reliability and validity of the scale, it only proves the internal structure of UOC. As a measuring scale, it lacks a certain norm, therefore, to further enlarge the research of big sample so as to set up a norm which is one of goals of the future researches.

\section{Acknowledgements}

This research was supported by National Social Science Foundation Grant (11XSH019) and the Fundamental Research Funds for the Central Universities (SWU1309371).

\section{REFERENCES}

Deng, W.-G., Pan, X.-F., \& He, H.-Y. (2006). Relationship between Interpersonal Climate of School and Mental Health of Teachers in Junior High School. Journal of Educational Science of Hunan Normal University, 5, 78-81

Owens, R. G., Miantao, S., et al. (1987). Organizational behavior in Education (p. 207). Central China Normal University' Press.

Ou, Z.-H., Pan, X.-F., \& Huang, H.-L. (2008). Relationship between Teachers Mental Health and Interpersonal Climate of their Universities. Chinese Mental Health Journal, 22, 341-343.

Pan, X.-F., \& Cheng, Z. F. (2001). An Investigation of the Current Situation of Applying Affective Factors in Teaching by Teachers in
Secondary Schools. Psychological Development and Education, 2, 59-64.

Pan, X. F., \& Sun, Y. L. (2002). A Study about the Reliability and Validity of the Organizational Climate for Secondary School Scale. Journal of Education Science of Hunan Normal University, 1, 123126.

Pan, X. F., Qin, Q. W., \& Tan, X. H. (2006). An Analysis of the Relationship between the School Organizational Climate and Teachers' Job Performance. Psychological Science, 29, 185-188.

Pan, X. F., \& Qin, Q. W. (2007). The Measurement of the Middle School OrganizationalClimate for its Relationship to Teachers' Mental Health. Psychological Science, 30, 982-986.

Zhou, S. X. (1998). Content and Measurement of School Climate. Journal of Sichuan College of Education, 14, 23-27

Tang, J., \& Chen, W. Q. (2001). From "Organizational Climate” to "Organizational culture"-Logic of Concept Development. Journal of Developments In Psychology, 2001, 62-65.

Tian, B., \& Li, L. (2006). A Research on the Relations between Involvement of Organizational Culture and Employee Satisfaction. Psychological Science, 29, 189-193.

Yin, J., \& Ma, S. C. (2009). Relationships among College Organization Atmosphere, Study condition and Study Achievement. China Journal of Health Psychology, 17, 38-41.

Zhu, G. Q. (1982). Zhu guangqian's aesthetic corpus (the first volume, pp. 62-71). Shanghai Literature \& Art Publishing House.

Cullen, K. W., et al. (1999). Influence of school organizational characteristics on the outcomes of a school health promotion program. Journal of School Health, 69, 76-380. http://dx.doi.org/10.1111/j.1746-1561.1999.tb06433.x

Dumay, X. (2009). Origins and Consequences of Schools' Organizational Culture for Student Achievement. Educational Administration Quarterly, 45, 523-555. http://dx.doi.org/10.1177/0013161X09335873

Gelade, G., \& Gilbert, P. (2003). Work climate and organizational effectiveness: The application of data envelopment analysis in organizational research. Organizational Research Methods, 6, 482-501. http://dx.doi.org/10.1177/1094428103257364

Gottfredson, G. D., et al. (2005). School climate predictors of school disorder: Results from a national study of delinquency prevention in schools. Journal of Research in Crime and Delinquency, 42, 412-444. http://dx.doi.org/10.1177/0022427804271931

Griffith, J. (2006). A compositional analysis of the organizational climate-performance relation: Public schools as organizations. Journal of Applied Social Psychology, 36, 1848-1880. http://dx.doi.org/10.1111/j.0021-9029.2006.00085.x

George, G. S. (1963). Characteristics of Intellectual Climate in College Environments. Harvard Educational Review, 31, 5-41

Halpin, A. W., \& Croft, D. B. (1963). The organizational climate of schools. Chicago: Midwest Administration Center of the University of Chicago.

Hoy, Wayne. K., TarterT, C. J., Bliss, J. R. (1990). Organizational-climate, school-health, and effectiveness: A comparative-analysis. Educational Administration Quarterly, 26, 260-279.

Hoy, W. K., John Tarter, C., Robert, B., \& Kottkamp (1991). Open school/Health school: measuring organizational climate. Sage Publications,

Hoy, W. K., \& Hannum, J. W. (1997). Middle school climate: An empirical assessment of organizational health and student achievement. Educational Administration Quarterly, 33, 290-311. http://dx.doi.org/10.1177/0013161X97033003003

Hoy, W. K. Hannum, J., \& Tstchannen-Moran, M. (1998). Organizational climate and student achievement: A parsimonious view. Journal of School Leadership, 8, 336-359.

Nalcaci, A. (2012). School climate in prediction of job satisfaction according to teacher perceptions. Energy Education Science and Technology Part B-social and Educational Studies, 4, 1441-1446.

Pan, X. F., \& Qin, Q. W. (2007). An Analysis of the relation between secondary school organizational climate and teacher job satisfaction. Chinese Education and Society, 40, 65-77.

Riehl, C., \& Sipple, J. W. (1996). Making the most of time and talent: 
Secondary school organizational climates, teaching task environments, and teacher commitment. American Educational Research Journal, 33, 873-901. http://dx.doi.org/10.3102/00028312033004873 Rensis, L., \& Jane, G. L. (1976). New Ways of Managing Conflict (pp. 218-219). New York: MeGraw-Hill.

Van Houtte, M. (2005). Climate or culture? A plea for conceptual clarity in school effectiveness research. School Effectiveness and School Improvement, 16, 71-89. http://dx.doi.org/10.1080/09243450500113977

Hoy, W. K., Hoffman, J., Sabo, D., \& Bliss, J. (1996). The organizational climate of middle schools: The development and test of the OCDQ-RM. Journal of Educational Administration, 34, 41-59. http://dx.doi.org/10.1108/09578239610107156

Zhu, C., Devos, G., \& Li, Y. F. (2011). Teacher perceptions of school culture and their organizational commitment and well-being in a Chinese school. Asian Pacific Education Review, 12, 319-328. 